\title{
Performance Comparison of RSS Algorithms for Indoor Localization in Large Open Environments
}

\author{
Nico Podevijn ${ }^{1}$, David Plets ${ }^{1}$, Jens Trogh ${ }^{1}$, Abdulkadir Karaagac ${ }^{2}$, \\ Jetmir Haxhibeqiri ${ }^{2}$, Jeroen Hoebeke ${ }^{2}$, Luc Martens ${ }^{1}$, Pieter Suanet ${ }^{3}$, Wout Joseph ${ }^{1}$ \\ ${ }^{1}$ Department of Information Technology, University of Ghent/IMEC, WAVES, Ghent, Belgium, nico.podevijn@ugent.be \\ ${ }^{2}$ Department of Information Technology, University of Ghent/IMEC, IDLab, Ghent, Belgium \\ ${ }^{3}$ Aucxis crba, Stekene, Belgium
}

\begin{abstract}
We develop and benchmark four RSS localisation algorithms where different a priori knowledge is required. The selection of the best algorithm depends on the availability of additional information on path loss exponent and/or transmit power. We compare our algorithms with centroid localization and show that the algorithms provide better results for shadowing on the values not exceeding 6dB. We perform experiments and simulations with Bluetooth Low Energy and LoRaWAN technologies and select the best technology and algorithm for localisation in large open industrial environments.
\end{abstract}

Index Terms-LoRA, BLE, RSS, Localization, Algorithm,open environment, industry

\section{INTRODUCTION}

Asset tracking in large industrial warehouses is mandatory to optimize the logistic supply chain processes. A localization solution is mostly a trade off between accuracy, delay, energy consumption, scalability and other metrics such as cost and implementation effort. Although many localization methods exist, we consider Received Signal Strength (RSS)-based localization since it is well suited for localization in zones where only coarse grained accuracy is needed. An important advantage of RSS is the fact there is no additional hardware cost since RSS values are mostly available on each hardware platform. Once RSS information is available from different anchors with a known location, an algorithm is needed to convert the RSS values to a location estimate. The novelties of the paper are threefold. Firstly, it describes 4 simple and effective algorithms for RSS-based localization. Secondly, it describes the best algorithm to use in line-of sight conditions. Finally, it compares experimentally Bluetooth Low Energy (BLE) and LoRa technologies in terms of achievable accuracy in a large open industrial hall. The paper is structured as follows. Section II describes related work on RSS-based algorithms. Section III describes our simple implemented methods for RSS-based localization. Section IV provides simulation results and discussion. In Section V experimental RSS models are described for Bluetooth Low energy and LoRa. The best technology is selected based on the results from Section IV. Finally, we conclude the paper in Section V.

\section{RELATED WORK}

Although not accurate, probably the most simple RSS algorithms are the centroid and proximity localisation methods [1]. The centroid algorithm maps the estimated location at

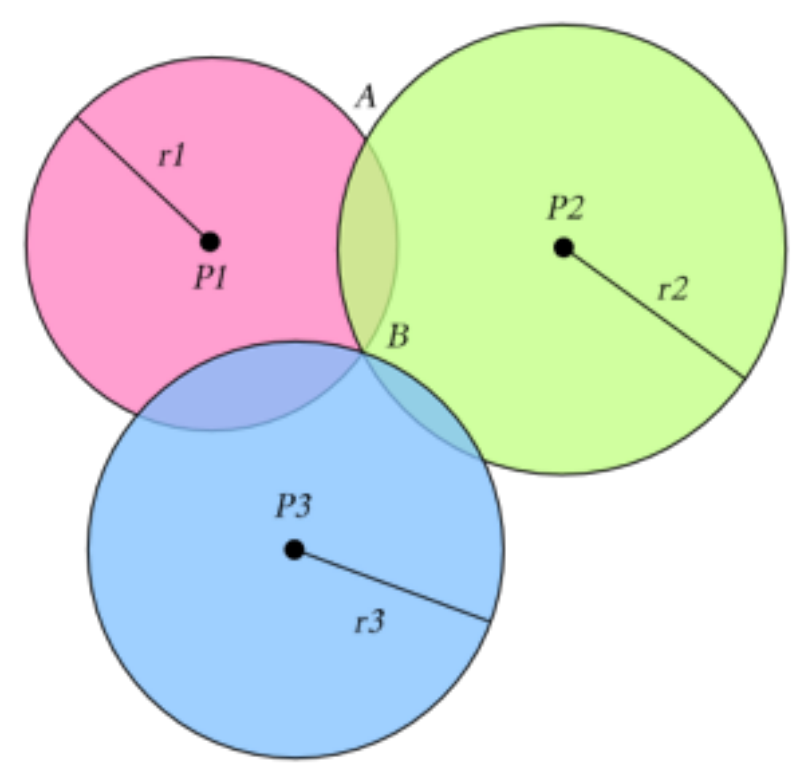

Fig. 1. Solution to the trilateration problem when the distances from the mobile node to the anchors are known.

the center of the base stations that received the packet. The proximity algorithm maps the location at the base station that received the packet with highest RSS value. In [2], a weighted centroid localisation algorithm is presented. It consists of a simple combination of centroid and proximity algorithms and takes into account the actual received RSS values. Although it can provide more accurate results than centroid or proximity algorithms, the weighting factor still needs to be determined. In [3], a straightforward algorithm is presented for cases where the distance is known from the anchors. The distance input can be estimated from the RSS values using a RSS model. Figure 1 shows the estimated location (B) when the distances $\mathrm{r} 1, \mathrm{r} 2$ and $\mathrm{r} 3$ to the anchors $\mathrm{P} 1, \mathrm{P} 2, \mathrm{P} 3$ are known. However, in practice the circles do not intersect due to inaccuracies in the distance estimations. Although [3] still can provide the most likely location, its implementation is complex. It depends on a correct RSS model and can give a solution which is not on the considered map. In [4], a method is described to perform multilateration without known transmit power and RSS model, i.e. without knowledge of the transmitting node 


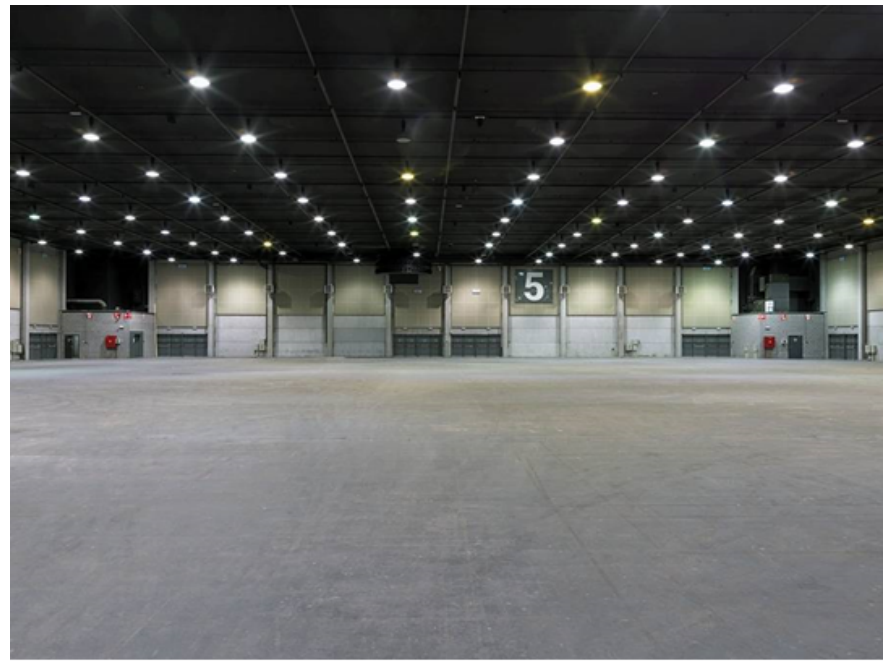

Fig. 2. Picture of the indoor open environment.

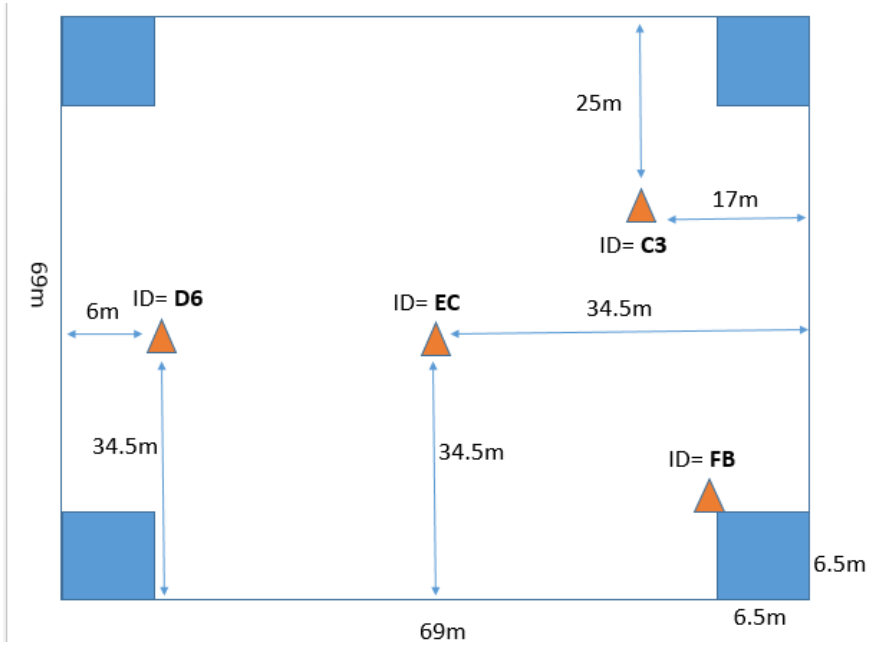

Fig. 3. BLE anchor placement (Triangles) for one-slope RSS model estimation in the indoor environment.

or the environment. The method provides a fairly simple mathematical framework but the analytical solution might not be within the considered map due to the fact that it considers all possible solutions in an infinite 2D space. An extensive overview and comparison of analytical RSS algorithms that provide more accurate localization can be found in [5]. Performing localisation in more complex environments is done in the work of [8]. Our work will provide simple low-information algorithms in large open environments.

\section{Materials AND Methods}

\section{A. Creation of one-slope RSS model}

Figure 2 shows a picture of the indoor open industrial environment where RSS measurements have been performed. These measurements will be used to benchmark the performance of different localization algorithms. In Figures 3 and 4, the floorplan of the indoor hall is shown. The BLE and LoRa

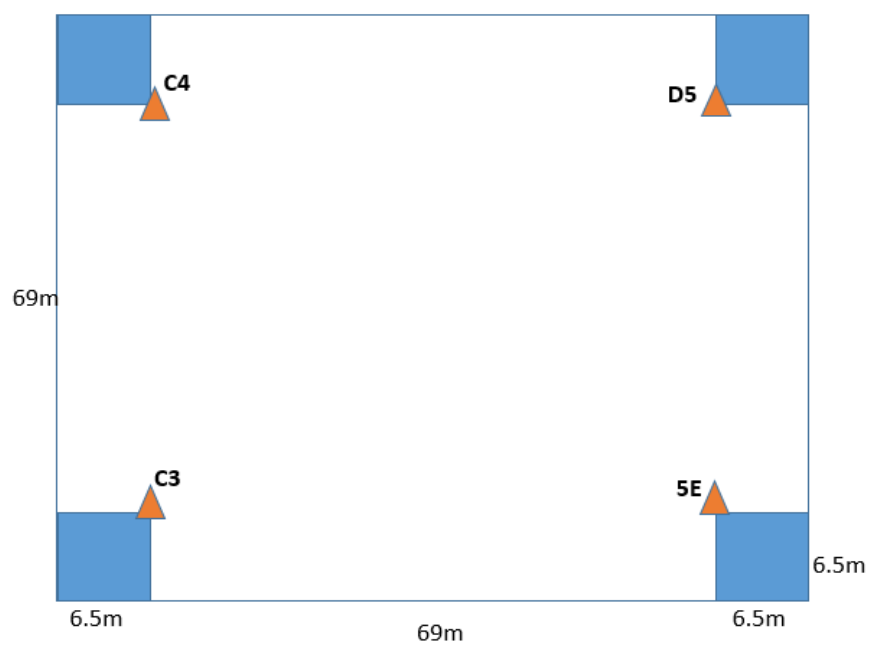

Fig. 4. LoRa anchor placement (Triangles) for one-slope RSS mode estimation in the indoor environment.

anchor locations are shown by triangles in Figures 3 and 4 respectively. The indoor environment is a large open space where line-of sight conditions (LOS) apply and measures $69 \mathrm{~m} \times 69 \mathrm{~m}$. We define 100 testpoints, homogeneously spaced $5 \mathrm{~m}$ apart in the test environment. At these 100 test locations we transmit LoRa [7] packets on spreading factor 7 (has the highest update rate) with a mobile node (LoRa scenario, Fig. 4) or receive BLE packets from the anchor nodes (BLE scenario, Fig. 3). Table I lists the used hardware to perform these experiments. All antennas were omnidirectional dipoles at $2.4 \mathrm{GHz}$ (BLE) and $868 \mathrm{MHz}$ (LoRa). During the measurements the influence of the body on the antennas was kept minimal [9]. Details of the configuration and settings can be found in Table III-A. Next, we record the RSS values together with the distance to the anchors on the test points. The final step is to plot the RSS values as a function of distance and perform a linear regression on the data in order to estimate the (one-slope RSS) model.

TABLE I

HARDWARE USED FOR THE EXPERIMENTS

\begin{tabular}{cc}
\hline \hline Technology & Type \\
\hline LoRa TX (Mobile Node) & Microchip LoRa Mote \\
LoRa RX (Gateway Anchor) & Multitech Conduit \\
BLE TX (Beacon Anchor) & AnkhMaway USB iBeacon \\
BLE RX (Mobile Node) & Nordic nRF52 DK \\
\hline
\end{tabular}

The RSS model is described as:

$$
R S S[d B m]=R S S_{0}-10 \cdot n \cdot \log 10(d)+X_{\sigma}
$$

The parameters to estimate are $R S S_{0}[d B m], n$, and $\sigma[d B]$ which denote the RSS value at distance $\mathrm{d}=1 \mathrm{~m}$, the path loss exponent and the standard deviation of the shadowing $X_{\sigma}[\mathrm{dB}]$ around the model, respectively.

\section{B. Localization Algorithms}

Four localization algorithms were developed. All four assume a different availability of information on the configura- 


\begin{tabular}{lcc} 
Parameter & BLE Value & LoRa Value \\
\hline Frequency band & $2.4 \mathrm{GHz}$ & $868 \mathrm{MHz}$ \\
Anchor height & $1.5 \mathrm{~m}$ & $1.5 \mathrm{~m}$ \\
Mobile node height & $2 \mathrm{~m}$ & $2 \mathrm{~m}$ \\
Radiation pattern & Omnidirectional & Omnidirectional \\
Transmit power & $18 \mathrm{dBm}$ & $14 \mathrm{dBm}$ \\
Antenna gains & $2 \mathrm{dBi}$ & $2 \mathrm{dBi}$ \\
Transmission & anchor to mobile node & mobile node to anchor \\
Packet RSS Averaging & yes (over 14-16 packets in 1.65s) & no (single packet) \\
\multicolumn{2}{c}{ ConfIGURATION AND SETTINGS FOR BLE AND LORA }
\end{tabular}

tion such as transmit power and path loss exponent as indicated in Table III. For example RSS(Ptx) only requires input of the transmit power Ptx of the transmitting node (or anchor). In what follows, we will describe the operation of the 4 algorithms. For all algorithms, the floorplan is considered and divided into grid points (100x100 points) of possible mobile locations.

TABLE III

LOCALIZATION ALGORITHMS AND DEPENDENCIES ON CONFIGURATION INFORMATION ( $\mathrm{N}=$ PATH LOSS EXPONENT, PTX = TRANSMIT POWER $)$

\begin{tabular}{ccc}
\hline \hline Algorithm & PL exponent & Transmit power \\
\hline RSS(n,Ptx) & Required & Required \\
RSS(Ptx) & - & Required \\
DRSS(n) & Required & - \\
DRSS() & - & - \\
\hline
\end{tabular}

1) RSS (Ptx,n) algorithm: During the offline phase, theoretical received RSS values are calculated according to the representative (measured) RSS model for each point of the grid. During the online phase the measured RSS values are 'compared' with the theoretical ones for each grid point. The grid point which has its theoretical RSS values closest (least squares) to the ones measured is determined as the estimated location $(\mathrm{X}, \mathrm{Y})$ :

$$
(X, Y)=\min _{x, y} \sum_{i=1}^{N}\left(R S S_{(x, y), i, T}-R S S_{i, M}\right)^{2}
$$

Here $R S S_{(x, y), i, T}$ denotes the theoretical RSS value at position (x,y) from or at anchor $\mathrm{i}$ (see eq. 1), $\mathrm{i}=1 . . \mathrm{N}$ with $\mathrm{N}$ the number of anchors. $R S S_{i, M}$ is the measured RSS value from or at anchor $\mathrm{i}$ and is therefore required. $R S S_{0}$ is obtained from a measurement at power Ptx.

2) RSS(Ptx) algorithm: This algorithm operates the same as $\operatorname{RSS}(\mathrm{Ptx}, \mathrm{n})$ but since the RSS model is unknown, it is estimated from the measured samples (for each location). The path loss exponent $n$ is first assumed to be equal to $n$ $=1$. Then RSS $(\operatorname{Ptx}, \mathrm{n})$ estimates an optimal location for that $\mathrm{n}$ (see first algorithm). The parameter $\mathrm{n}$ is then increased up to 6 in steps of 0.2. After these iterations a set of optimal locations are found for each iteration of $n$. For each location we calculate the cost function (as in [6]), which determines for which location and corresponding path loss exponent the one-slope model gives the best fit:

$$
n_{\text {opt }}=\arg \min _{n} \sum_{i=2}^{N}\left[\left(d_{1, n} / d_{i, n}\right)-\left(P_{i} / P_{1}\right)^{(1 / n)}\right]
$$

here $d_{i, n}$ and $P_{i}$ denote the distance to the $\mathrm{i}$-th anchor and received power respectively. The latter is converted from the RSS value in $\mathrm{dBm}$ to a power unit in watts. $\mathrm{N}$ is the total number of anchors and $n_{\text {opt }}$ the estimated path loss exponent.

3) DRSS(n) algorithm: This Differential RSS algorithm operates without prior knowledge of the transmit power. All RSS vales are normalized to the strongest RSS value effectively eliminating Ptx (and $R S S_{0}$ ) from the model. The operation is then similar to the first algorithm RSS(Ptx,n) but with RSS replaced by DRSS which is obtained by:

$$
D R S S_{i}=R S S_{i}-R S S_{1}
$$

Where $R S S_{1}$ denotes the strongest received signal strength. In the algorithm, $\mathrm{n}$ is considered to be constant and known.

4) DRSS() algorithm: This Differential RSS algorithm operates without information on path loss exponent or transmit power. Its operation is a combination of the $2^{\text {nd }}$ and $3^{\text {rd }}$ algorithm. First all possible DRSS values are calculated and stored in a database for path loss exponent $n=1$. The measured RSS values are converted to DRSS and a best match is searched through the database. This location is stored and the process is repeated for all $\mathrm{n}$. Then, the location which best bests the one slope model with the corresponding $\mathrm{n}$ is taken as the estimated location.

The algorithms can be used on experimental or simulated data. For the simulation we consider the same 100 testpoint locations from our measurement campaign and calculate the theoretical RSS values from/to the anchors using a one-slope RSS model using eq. (1). All our processing was done in MATLAB.

\section{C. $k$-NN applied to the algorithms}

In [10] a possible well known localization improvement is suggested. The idea is to estimate multiple $(\mathrm{k})$ nearest neighbor 
(NN) solutions instead of a single (NN) one. After the multiple solutions are found they are weighted and a final solution is obtained. The k-NN method is applied to our $\operatorname{RSS}(\mathrm{Ptx}, \mathrm{N})$ algorithm with $\mathrm{k}=4$.

\section{Performance for different $\sigma$ values}

The parameter that affects the localization accuracy the most is the (fixed) $\sigma$ parameter. The higher this value the more variation on the RSS and therefore the error on the localisation estimate gets larger. To quantify the impact of this parameter on the p50 en p90 accuracy metrics a simulation (100 testpoints, 30 simulations per testpoint) is carried out. $\sigma$ is varied from 1 to $10 \mathrm{~dB}$ in steps of $1 \mathrm{~dB}$. At each step the performance of the 4 algorithms is evaluated. For comparison, the centroid method is also simulated.

\section{RESUlts}

\section{A. Experimental one-slope RSS model}

RSS data was collected from 100 testpoints in a 10x10 grid with testpoints spaced $5 \mathrm{~m}$ apart in the environment. For BLE, 14 samples were collected per testpoint per anchor during a period of $1.65 \mathrm{~s}$. For LoRa only 1 sample per testpoint per anchor was collected. Figure 5 shows the RSS variation over distance from each anchor for BLE without averaging. The combined estimated BLE RSS model parameters are $R S S_{0}=$ $-26 \mathrm{dBm}, \mathrm{n}=1.4$ and $\sigma=5.2 \mathrm{~dB}$. Figure 6 shows the RSS model in case the averaged samples are fit. The combined estimated BLE RSS model parameters are now $R S S_{0}=-26$ $\mathrm{dBm}, \mathrm{n}=1.2$ and $\sigma=3.2 \mathrm{~dB}$. The averaging therefore helps to reduce the overall variation around the model which will lead to better localisation results.

For LoRa the RSS model parameters are $R S S_{0}=-23 \mathrm{dBm}$, $\mathrm{n}=1.5$ and $\sigma=6 \mathrm{~dB}$. The model is shown in Fig. 7. Note that for LoRa the term Equivalent Signal Power (ESP) is equivalent to RSS if the signal to noise ratio is greater than $1(0 \mathrm{~dB})$. The variation $(\sigma)$ around the model is also much smaller for BLE then for LoRa because BLE allows for averaging over (14-16) different transmissions in a short period of time (only $1.65 \mathrm{~s}$ ), which is not possible for LoRa (One sample each $5 \mathrm{~s})$. Therefore, BLE will obtain better localisation results then LoRa for the same setup. When comparing all RSS models with free space we note that free space underestimates the RSS model at short distances and overestimates the RSS model at larger distances. The real RSS model can be approximated by the free space RSS model only if an error of up to $6 \mathrm{~dB}$ is allowed.

\section{B. Performance of algorithms}

The performance of the localisation using the algorithms is shown for BLE in Figure 8. The performance was assessed for both experimental and simulated data from 100 testpoints spaced $5 \mathrm{~m}$ apart. For the simulation, we calculate the theoretical RSS values from/to the anchors using a one-slope RSS model using eq. (1). Table IV lists the obtained accuracy metrics (50 and 90 percentiles). All 4 algorithms have similar performance and that simulated performance agrees well with

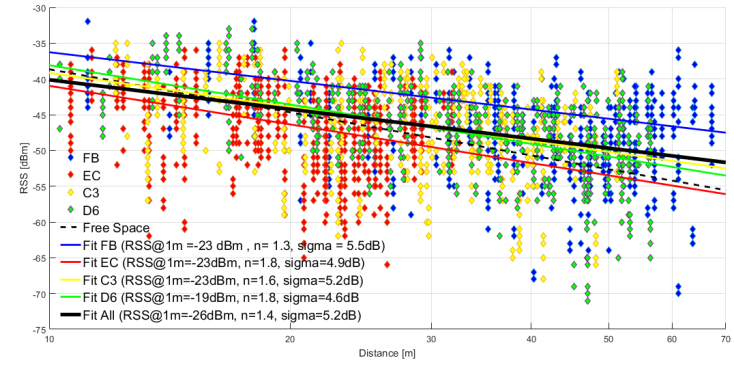

Fig. 5. Experimental RSS model for BLE without averaging. FB, EC, C3 ,D6 are the samples collected on the different anchors

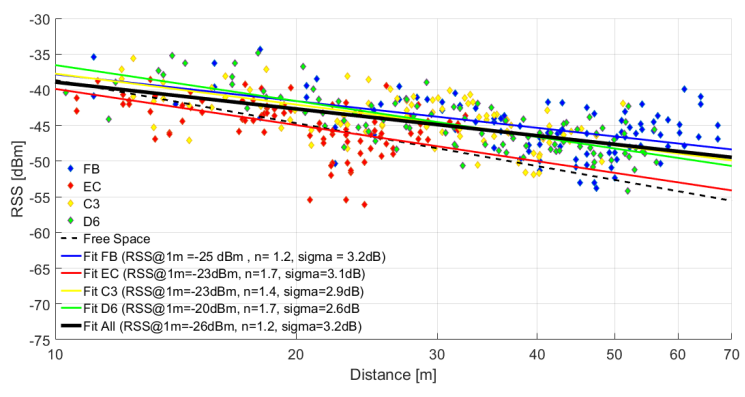

Fig. 6. Experimental RSS model for BLE with averaging.FB, EC, C3 ,D6 are the RSS samples collected on the different anchors

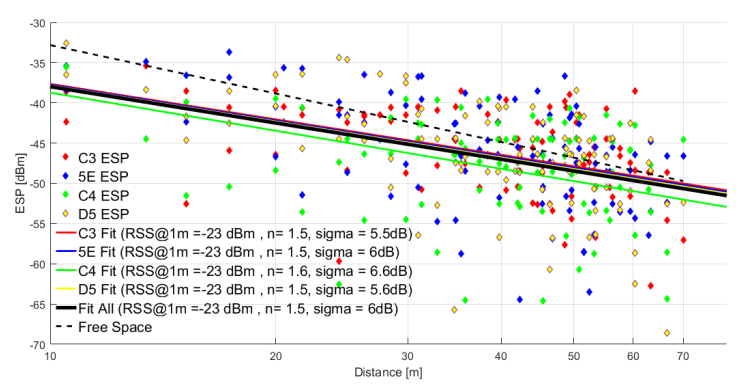

Fig. 7. Experimental RSS model for LoRa .C3, 5E, C4, D5, are the Equivalent Signal Power (ESP) samples collected on the different anchors

the measurement. Even without prior knowledge of transmit power and path loss exponent (DRSS()), the median error is $17 \mathrm{~m}$ and is still better than the median of the centroid localisation $(20 \mathrm{~m})$.

Figure 9 and Table $\mathrm{V}$ show the localisation results for LoRa. Again the measurements are in agreement with the simulations. The obtained experimental median error is around 19$22 \mathrm{~m}$ for our algorithms and the centroid median error is $19 \mathrm{~m}$. We note that the centroid provides better results than our algorithms. This is due to the high fluctuations of RSS values as seen in the RSS model (high $\sigma$ values) and no averaging possibility on the samples. Therefore, LoRa RSS is not suited well for indoor localisation in open environments. Since BLE provides better results than LoRa and due to the fact its cost is also lower per anchor, it is chosen as the preferred technology for indoor localization in the considered industrial open hall. 


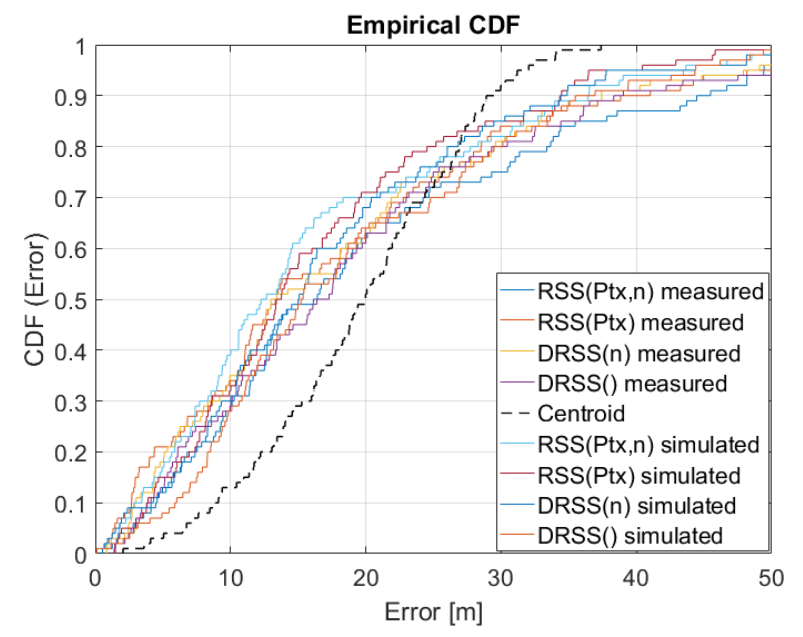

Fig. 8. Localisation accuracy for BLE when considering the algorithms for both the measured and simulated case .

TABLE IV

LOCALISATION ACCURACY RESULTS FOR BLE. THE SUBSCRIPTS S AND M DENOTE SIMULATION AND MEASUREMENT RESPECTIVELY FOR THE 50 AND 90 PERCENTILE ERRORS

\begin{tabular}{ccccc}
\hline \hline Algorithm & $p_{50}^{s}[m]$ & $p_{50}^{m}[m]$ & $p_{90}^{s}[m]$ & $p_{90}^{m}[m]$ \\
\hline RSS(Ptx,n) & 12 & 16 & 36 & 44 \\
RSS(Ptx) & 13 & 13 & 34 & 38 \\
DRSS(n) & 15 & 13 & 34 & 37 \\
DRSS() & 15 & 17 & 36 & 37 \\
Centroid & 20 & 20 & 29 & 29 \\
\hline
\end{tabular}

TABLE V

LOCALISATION ACCURACY RESULTS FOR LORA. THE SUBSCRIPTS S AND M DENOTE SIMULATION AND MEASUREMENT RESPECTIVELY FOR THE 50 AND 90 PERCENTILE ERRORS

\begin{tabular}{ccccc}
\hline \hline Algorithm & $p_{50}^{s}[m]$ & $p_{50}^{m}[m]$ & $p_{90}^{s}[m]$ & $p_{90}^{m}[m]$ \\
\hline RSS(Ptx,n) & 25 & 19 & 44 & 41 \\
RSS(Ptx) & 28 & 22 & 45 & 42 \\
DRSS(n) & 25 & 22 & 47 & 39 \\
DRSS() & 24 & 22 & 47 & 39 \\
Centroid & 19 & 19 & 28 & 28 \\
\hline
\end{tabular}

\section{Performance of $k-N N$ when applied to $\operatorname{RSS}(P t x, n)$}

Figure 10 shows the performance of the RSS(Ptx,n) with and without applying the k-NN method for both the simulation and experimental case. From the figure it is clear k-NN does not improve the obtained accuracy. This is due to the fact our fingerprint map was very dense (50x50 points) with almost identical $\mathrm{k}$ neighbor points in this open environment. Weighted $\mathrm{k}-\mathrm{NN}$ therefore has similar performance as NN [10].

\section{Performance for different $\sigma$ values}

Figures 11 and 12 show the p50 and p90 accuracy simulation results repectively for different $\sigma$ values. We clearly see all 4 algorithms have similar performance and provide better results than centroid when $\sigma$ is relatively small. For high $\sigma$ ( $\sigma>7 \mathrm{~dB}$ for $\mathrm{p} 50, \sigma>4.5 \mathrm{~dB}$ for $\mathrm{p} 90$ ) we see that centroid provides better results. Therefore it is advisable to estimate $\sigma$ in the environment in advance before selecting an algorithm.

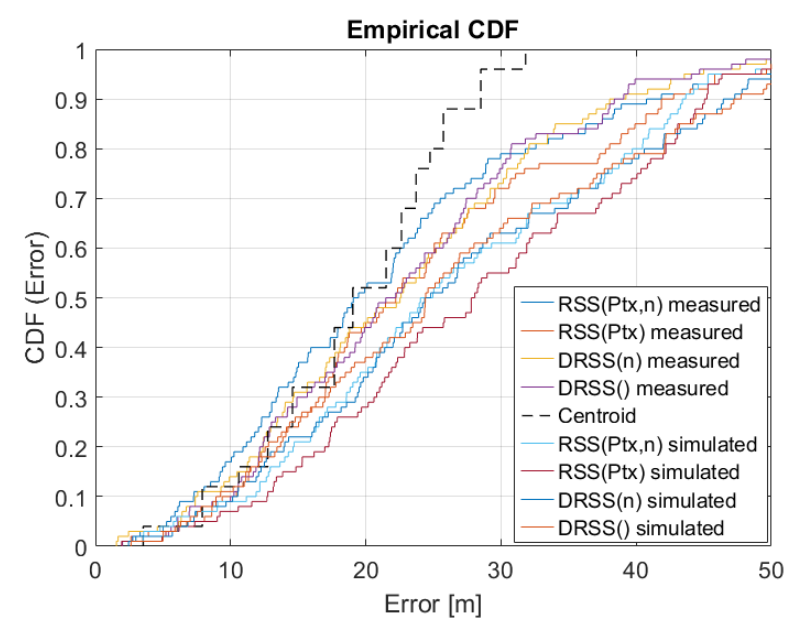

Fig. 9. Localisation accuracy for LoRa when considering the algorithms for both the measured and simulated case.

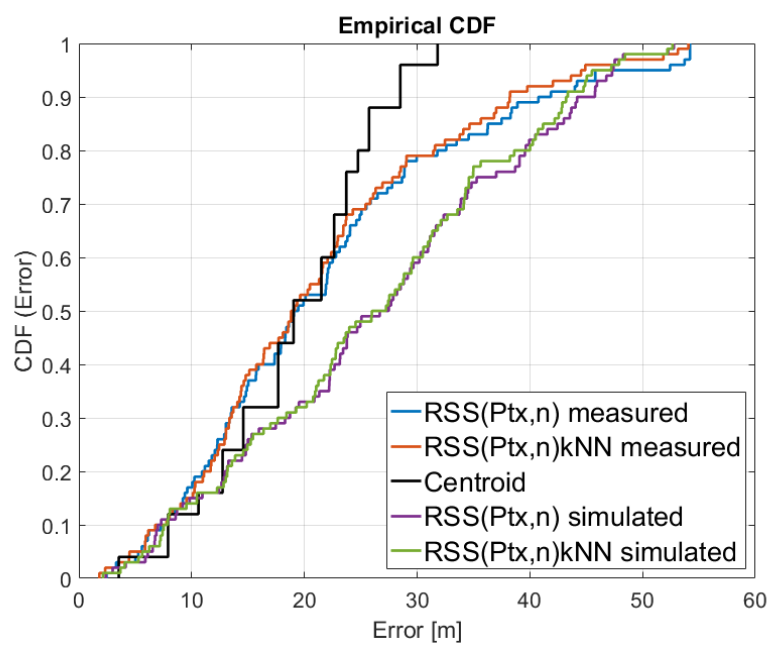

Fig. 10. Localisation accuracy with and without applying the k-NN method

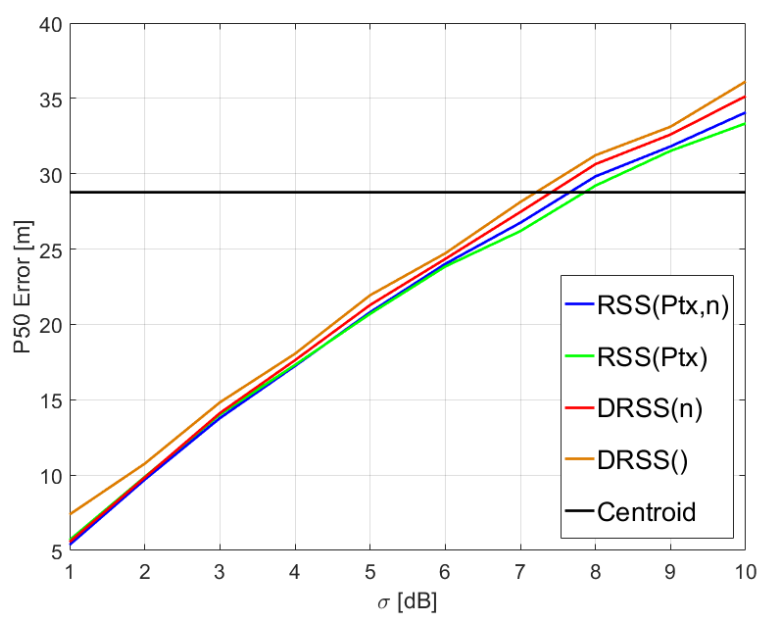

Fig. 11. P50 localization accuracy under varying $\sigma$. 


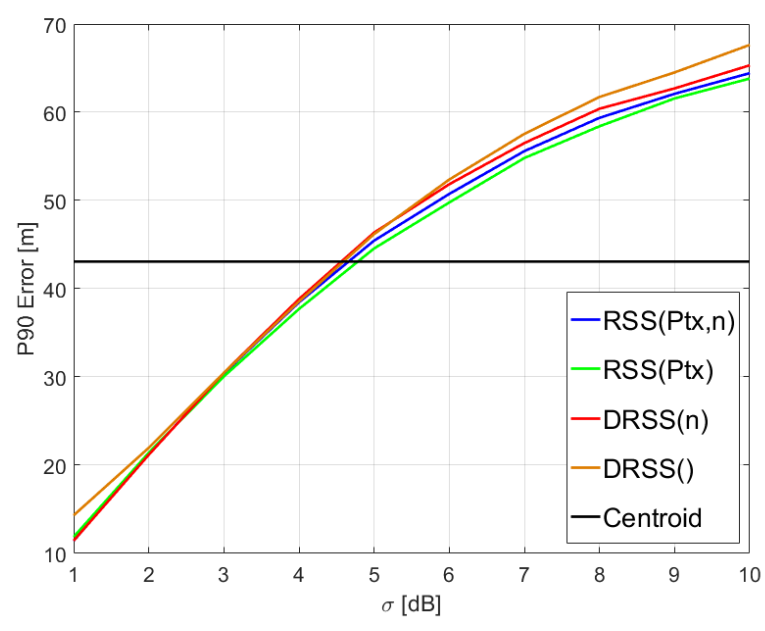

Fig. 12. P90 localization accuracy under varying $\sigma$.

\section{CONCLUSION}

In this paper we developed 4 simple low-information localization algorithms. We applied the algorithms to the special case of a large indoor indoor environment for which we experimentally obtained a one-slope RSS model. The preferred algorithm does not rely on additional information such as transmit power and path loss exponent. We compared BLE and LoRa technologies. The preferred technology turned out to be BLE due to its lower cost and averaging capabilities. Considering our configuration when using BLE, we obtained a median accuracy of $15 \mathrm{~m}$ with just 4 BLE beacons placed in an environment measuring $69 \mathrm{~m} \mathrm{x} 69 \mathrm{~m}$. There was good agreement between the obtained measurements and simulation localization results. Further work includes the investigation of the improvement of the accuracy by placing more anchors and determining their optimal location. Finally, the sensitivity of the algorithms to the correctness of the provided RSS model and/or transmit power is part of future work.

\section{ACKNOWLEDGEMENT}

This research is part of the HYCOWARE project realized in collaboration with IMEC. Project partners are Egemin, Aucxis and Intation, with project support from VLAIO (Flanders Innovation and Entrepreneurship).

\section{REFERENCES}

[1] Orujov, Farid and Rytis Maskeliunas. Comparative Analysis of the Indoor Positioning Algorithms using Bluetooth Low Energy Beacons. (2016).

[2] A. Giorgetti, K. Magowe and S. Kandeepan, "Exact analysis of weighted centroid localization,” 2016 24th European Signal Processing Conference (EUSIPCO), Budapest, 2016, pp. 743-747.

[3] Norrdine, Abdelmoumen. (2015). An Algebraic Solution to the Multilateration Problem. 10.13140/RG.2.1.1681.3602.

[4] Y. Xu, J. Zhou and P. Zhang, "RSS-Based Source Localization When Path-Loss Model Parameters are Unknown," in IEEE Communications Letters, vol. 18, no. 6, pp. 1055-1058, June 2014.

[5] Y. Hu and G. Leus, "Robust Differential Received Signal Strength-Based Localization," in IEEE Transactions on Signal Processing, vol. 65, no. 12, pp. 3261-3276, June15, 152017.
[6] Y. Xu, J. Zhou and P. Zhang, "RSS-Based Source Localization When Path-Loss Model Parameters are Unknown,” in IEEE Communications Letters, vol. 18, no. 6, pp. 1055-1058, June 2014

[7] N.Podevijn, D.Plets, J.Trogh, L.Martens, P.Suanet, K.Hendrikse, and W.Joseph, "TDoA-based Outdoor Positioning with Tracking Algorithm in a Public LoRa Network." in Wireless Communications and Mobile Computing

[8] J. Trogh, D. Plets, L. Martens and W. Joseph, "Advanced indoor localisation based on the Viterbi algorithm and semantic data," 2015 9th European Conference on Antennas and Propagation (EuCAP), Lisbon, 2015, pp. 1-3.

[9] J. Trogh, D. Plets, A. Thielens, L. Martens and W. Joseph, ”Enhanced Indoor Location Tracking Through Body Shadowing Compensation," in IEEE Sensors Journal, vol. 16, no. 7, pp. 2105-2114, April1, 2016.

[10] V. Honkavirta , "Location fingerprinting methods in wireless local area networks". Master of science thesis Tampere University of Technology. 2008 . 